\title{
Etude comparative des caractéristiques morphobiométriques des chèvres Rousse et Noire de Maradi au Niger: analyse des paramètres quantitatifs et qualitatifs
}

\author{
Malam Gadjimi ADAM KADE ${ }^{1 *}$, Mamman MANI², Guiguigbaza-Kossigan DAYO ${ }^{3,4}$ et \\ Hamani MARICHATOU ${ }^{1}$
}

\author{
${ }^{I}$ Faculté d'Agronomie, Université Abdou Moumouni Dioffo - BP: 10662 Niamey, Niger. \\ ${ }^{2}$ Institut National de la Recherche Agronomique du Niger - BP: 429 Niamey, Niger. \\ ${ }^{3}$ Centre International de Recherche-Développement sur l'Elevage en zone Subhumide (CIRDES), \\ BP: 454 Bobo-Dioulasso 01, Burkina Faso. \\ ${ }^{4}$ Institut du Sahel (INSAH), Bamako, Mali. \\ *Auteur correspondant; E-mail: agadjimi@yahoo.com; Tél: (+227) 96532116 ou (+227) 93936353
}

\section{RESUME}

Le Niger, compte environ 43 millions de têtes de bétail dont $36 \%$ de caprins. La chèvre Rousse de Maradi se distingue par ses remarquables performances zootechniques. La chèvre Noire partageant le même biotope est discriminée. La présente étude a pour objectif de comparer les caractéristiques morphobiométriques de la chèvre Rousse de Maradi et sa variante Noire vivant dans la même zone agro-écologique. Ainsi, 144 caprins ont été choisis au hasard dans 11 villages/sites répartis dans 5 départements de la région de Maradi. Les caractères quantitatifs mesurés étaient : hauteur au garrot et à la croupe, tour de poitrine, longueur du corps, des oreilles, du cou et des cornes, largeur du bassin et profondeur de la poitrine. Les caractères qualitatifs appréciés étaient: ports d'oreilles, cornes, barbiche et pampille, longueur et type de poils et patrons pigmentaires. Il est ressorti qu'il n'existe aucune différence significative au seuil de 5\% entre les deux types pour tous les paramètres quantitatifs sauf pour la longueur des cornes. Pour les traits qualitatifs, la chèvre Rousse possède majoritairement des oreilles dressées $(87,9 \%)$, absence de barbiche et pampille, pelage ras et lisse. On note une absence de barbiche et pampille, des poils ras et lisses, un profil de tête rectiligne chez les deux types de caprins tandis qu'on observe des cornes ibex chez la Rousse et des cornes markhar chez la Noire. Cette étude a montré que les robes rousses et noires de la chèvre de Maradi ne constituent qu'une diversité dans la même race caprine.

(C) 2019 International Formulae Group. All rights reserved.

Mots clés: Chèvre Rousse, chèvre Noire, caractéristiques morphobiométriques, Maradi, Niger.

\section{Comparative study of morphobiometric characteristics of Red and Black Maradi goats in Niger: analysis of quantitative and qualitative parameters}

\begin{abstract}
Niger has about 43 million head of farm animals and goats numerically represent $36 \%$. The Red goat of Maradi is appreciated for its outstanding zootechnical performances compared to its Black variant. The objective of this study is to compare the morphobiometric characteristics of the red goat Maradi and its black variant sharing the same agro-ecological zone. Thus, 144 goats were randomly selected in 11 villages/sites
\end{abstract}


located in 5 departments of Maradi administrative region. Quantitative parameters measured were: Height at the withers and rump, Chest circumference, Body length, ears, neck and horns, Basin Width and Chest Depth. The qualitative characteristics appreciated were: ears, horns, goatee and tassel, length and type of hair and pigment patterns. It was found that there is no significant difference at the 5\% threshold between the two types for all quantitative parameters except for horn length. For all quantitative parameters, no significant difference was found at 5\% threshold between Red and Black goat of Maradi except horn length. For qualitative traits, the red goat has mostly erect ears (87.9\%). For both types of goats, there is an absence of goatee and tassel, short and smooth hairs, a straight head profile. It was noted ibex horns in Red goat and markhar horns in the Black goat. This study showed that the Red and Black coat colors of the Maradi goat are variants of a same goat breed.

(C) 2019 International Formulae Group. All rights reserved.

Keywords: Red goat, Black goat, morphobiometric characteristics, Maradi, Niger.

\section{INTRODUCTION}

Au Niger, l'élevage représente environ $11 \%$ du PIB national et $25 \%$ du PIB agricole (Niger, 2014). Le cheptel est estimé à près de 43 millions de têtes (Niger, 2016) et l'espèce caprine y représente numériquement $36 \%$. La chèvre est rencontrée dans toutes les zones climatiques du Niger et son élevage occupe toute la population aussi bien sédentaire que nomade. Comme partout en Afrique de l'Ouest, deux groupes de chèvres sont distingués. Au Nord, les chèvres du Sahel sont de grande taille, le corps allongé, les membres longs et fins, le poil ras. Les chèvres naines du Sud sont de taille petite ou moyenne, le profil de la tête est rectiligne ou légèrement concave, le corps trapu, les membres courts et musclés, le poil ras.

Parmi les caprins du Niger, la chèvre Rousse de Maradi a focalisé plus d'attention. Déjà en 1933, sa diffusion a commencé par la castration de tous les boucs non roux. Après l'indépendance en 1960, la castration s'accompagnait des mesures législatives très sévères (interdiction d'abattage des femelles rousses, éliminations des chèvres bariolées et communes, etc.) et de la distribution des caprins Roux aux populations locales (Djariri, 2006). En 1965, le centre d'élevage caprin fut créé avec pour objectifs principaux la sélection et la diffusion de la chèvre Rousse de Maradi. Elle semble avoir son berceau dans le Sud des régions de Maradi (départements de Tessaoua, Aguié, Madarounfa, Guidan Roumdji et Commune de Maradi) et de Zinder (Matameye et Magaria), dans un espace couvrant environ $4000 \mathrm{~km}^{2}$ de part et d'autre de la ville de Tessaoua qui paraît en être le centre. Dans cet espace, la chèvre Rousse et sa variante à robe noire représentent plus de $70 \%$ des effectifs caprins (Djariri, 2006).

Les études publiées sur la chèvre Rousse de Maradi sur les aspects ethnologiques (Adigwe et Fayemi, 2005), les caractéristiques morphobiométriques et zootechniques (Marichatou et al., 2002; Marichatou et al., 2012) n'ont pas pu établir que les chèvres Rousse et Noire sont deux types caprins similaires à tous points de vue.

La présente étude est une première étape d'une série d'investigations qui vise à comparer les caractéristiques morphobiométriques aussi bien quantitatives que qualitatives des deux types de chèvres Rousse et Noire de Maradi.

\section{MATERIEL ET METHODES}

\section{Aire géographique de l'étude}

La région de Maradi (berceau de la chèvre Rousse) où est conduite cette étude est située au Centre-Sud du Niger (Figure 1). Elle couvre une superficie de $38500 \mathrm{~km}^{2}$ soit $3 \%$ du territoire national. Elle est limitée par la région de Zinder à l'Est, celles de Tahoua à l'Ouest, Agadez au Nord, et partage une frontière d'environ $150 \mathrm{~km}$ avec la République Fédérale du Nigeria au Sud. Son climat est de type sahélien avec une pluviométrie variant de 200 à plus de $600 \mathrm{~mm}$ par an du Nord au Sud (Niger, 2013). Les écarts de température et d'humidité y sont très élevés. 
Son environnement est caractérisé par un paysage de savane arborée ou arbustive, de plus en plus dégradée par l'extension des cultures et leur remontée vers le Nord. Le relief y est constitué par un vaste plateau du continental intercalaire légèrement incliné du Sud $(550 \mathrm{~m})$ au Nord $(400 \mathrm{~m})$. Les pentes et les plateaux dunaires, au sol léger, sont recouverts d'une brousse épineuse où dominent Bauhinia sp., Balanites sp., Combretum sp. et Acacia sp., en particulier Acacia albida et Acacia nilotica dont les feuilles sont appréciées des animaux. La région renferme un réseau hydrographique important de trois grands bassins: le Goulbi de Maradi, le Goulbi N'Kaba et la vallée de la Tarka. Ce réseau n'est fonctionnel que dans la partie méridionale de la zone (Goulbi de Maradi et Sud du Goulbi N'Kaba), le reste du réseau étant fossile (vallée de la Tarka et partie Nord du Goulbi N'Kaba).

L'étude a été conduite dans la bande Sud de cette région, notamment les départements de Guidan Roumdji, Aguié, Tessaoua, Madarounfa et la ville de Maradi, zone où cohabitent les deux types caprins. Elle a concerné onze sites (villages) qui ont été géo-référencés à l'aide d'un GPS (Global Positioning System) et se situent entre $13^{\circ} 75^{\text {, }}$ à $13^{\circ} 23^{\prime}$ de longitude Nord et $07^{\circ} 99^{\prime}$ à $07^{\circ} 08^{\prime}$ de latitude Est pour une altitude variant de 314 à $429 \mathrm{~m}$.

\section{Echantillonnage des animaux}

$\mathrm{Au}$ total, 144 caprins majoritairement adultes, âgés de plus d'un (1) an à quatre (4) ans $(33,3 \%)$ et plus $(66,6 \%)$ de robes Rousse (50\%), Noire $(47,22 \%)$ et composée (rousse et noire) $(2,78 \%)$; de femelles $(84,03 \%)$ et de mâles $(15,97 \%)$ ont fait l'objet de mensurations corporelles et d'observations qualitatives sur les 11 sites dont dix exploitations paysannes et un centre caprin dans la ville de Maradi. Le Tableau 1 présente la répartition de l'échantillon de l'étude par département, sexe et couleur de la robe.

\section{Collecte des données}

Sur le plan quantitatif, neuf paramètres barymétriques ont été mesurés sur chaque animal selon le guide de l'UA-BIRA (2019) et sur la base des études de Karimou (2015) et Mani et al. (2013). Il s'agit de: Hauteur au Garrot (HG), Tour de Poitrine (TP), Longueur du Corps (LCP), Largeur du Bassin (LB), Hauteur à la croupe $(\mathrm{HC})$, Profondeur de Poitrine (PP), Longueur des Oreilles (LO), Longueur du Cou (LCU) et Longueur des Cornes (LCR). Les mensurations ont été faites à l'aide du mètre ruban en ce qui concerne TP, $\mathrm{LCP}, \mathrm{LO}, \mathrm{LCU}$ et $\mathrm{LCR}$ et la règle métrique pour $\mathrm{HG}, \mathrm{PP}$ et $\mathrm{HC}$. La LB a été mesurée à l'aide d'un compas de tableau et d'une règle à triple décimètre. Toutes les mensurations ont été faites dans les conditions naturelles dans la journée à un intervalle de températures tolérables pour les animaux ( 23 à $33{ }^{\circ} \mathrm{C}$ ). La Figure 2 illustre ces différentes mensurations.

Les observations qualitatives ont concerné huit paramètres qui ont été appréciées selon 26 modalités et suivant le sexe et la couleur de la robe. Ces variables sont: port des oreilles, des cornes, de barbiche, de pampilles; longueur et type de poils; profil de la tête et patron pigmentaire.

Des fiches de collecte de données ont permis de répertorier toutes les informations de mesures et d'observations qualitatives.

\section{Analyse statistique}

Les données collectées ont été saisies sur une maquette élaborée à cet effet dans un tableur EXCEL 2010. Les analyses statistiques ont été effectuées avec les logiciels SPSS 17.0, XLSTAT 2014 et MINITAB 14. Le logiciel SPSS 17.0 a été utilisé pour les analyses descriptives. Les données quantitatives ont été exprimées sous forme de moyennes, écart-types et valeurs extrêmes et les données qualitatives sous forme de fréquences et effectifs. Les tests de comparaison de moyennes (ANOVA et test-t) ont été conduits avec le logiciel MINITAB 14. Le seuil de significativité retenu est de 5\%. Des analyses multi-variées, analyses en composantes principales (ACP) et analyses en composantes multiples (ACM) ont été réalisées avec le logiciel XLSTAT 2014 pour respectivement apprécier les corrélations entre les variables quantitatives et qualitatives. 


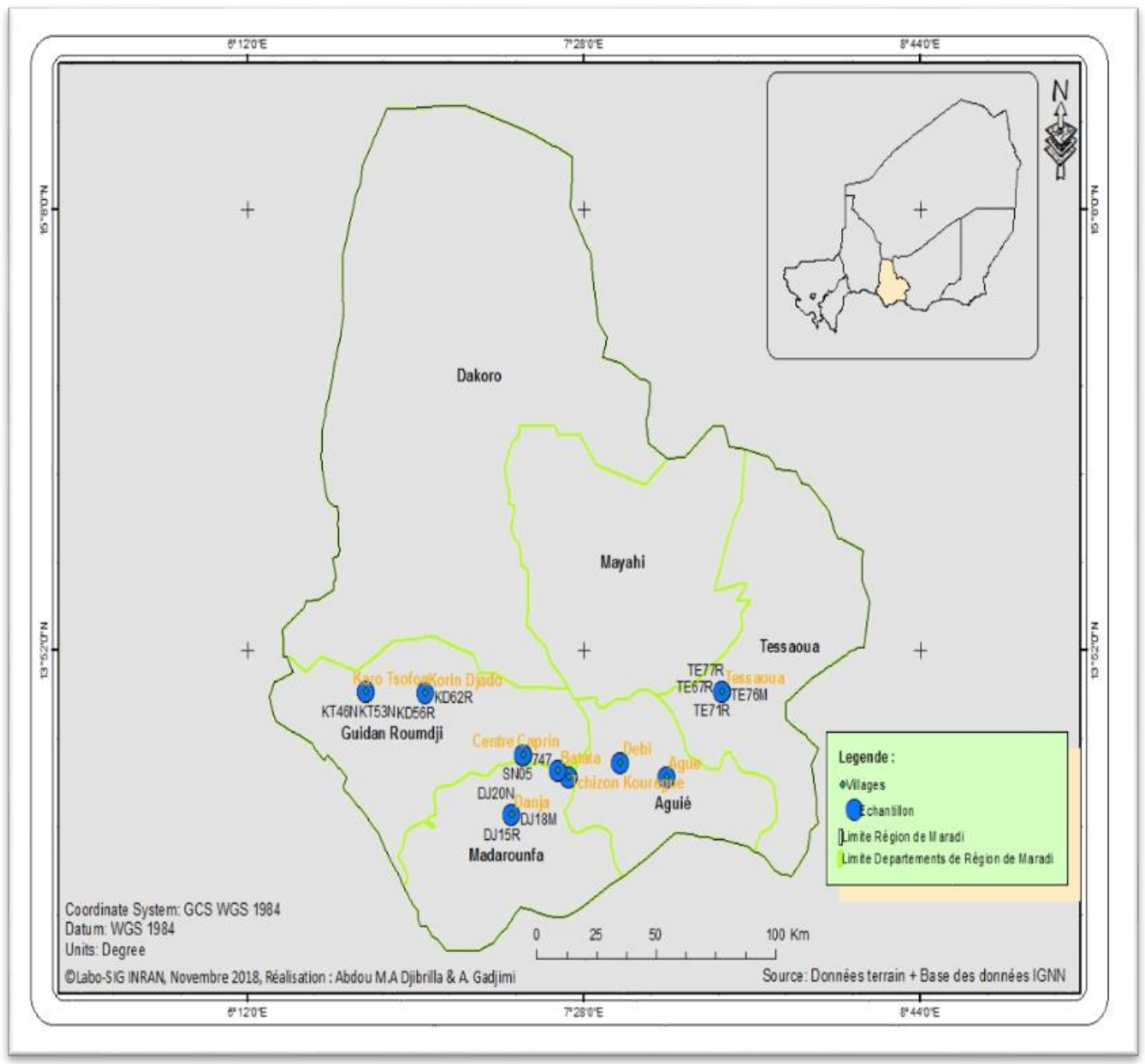

Figure 1: Sites de l'étude dans la région de Maradi au Niger.

Tableau 1: Distribution de l'échantillonnage de la population animale expérimentale.

\begin{tabular}{lccccccc}
\hline \multirow{2}{*}{ Département } & \multicolumn{2}{c}{ Echantillon } & \multicolumn{2}{c}{ Effectif par sexe } & \multicolumn{2}{c}{ Effectif par couleur de robe } \\
\cline { 2 - 7 } & $\begin{array}{c}\text { Nombre de } \\
\text { sites }\end{array}$ & $\begin{array}{c}\text { Nombre } \\
\text { d'individus }\end{array}$ & Femelle & Mâle & Rousse & Noire & Composée \\
\hline Aguié & 2 & 20 & 19 & 1 & 12 & 8 & 0 \\
Guidan & 2 & 25 & 25 & 0 & 13 & 11 & 1 \\
Roumdji & 4 & 38 & 36 & 2 & 7 & 30 & 1 \\
Madarounfa & 2 & 27 & 17 & 10 & 25 & 2 & 0 \\
Maradi ville & 1 & 34 & 24 & 10 & 15 & 15 & 4 \\
Tessaoua & $\mathbf{1 1}$ & $\mathbf{1 4 4}$ & $\mathbf{1 2 1}$ & $\mathbf{2 3}$ & $\mathbf{7 2}$ & $\mathbf{6 6}$ & $\mathbf{6}$ \\
\hline TOTAL & & & & & &
\end{tabular}




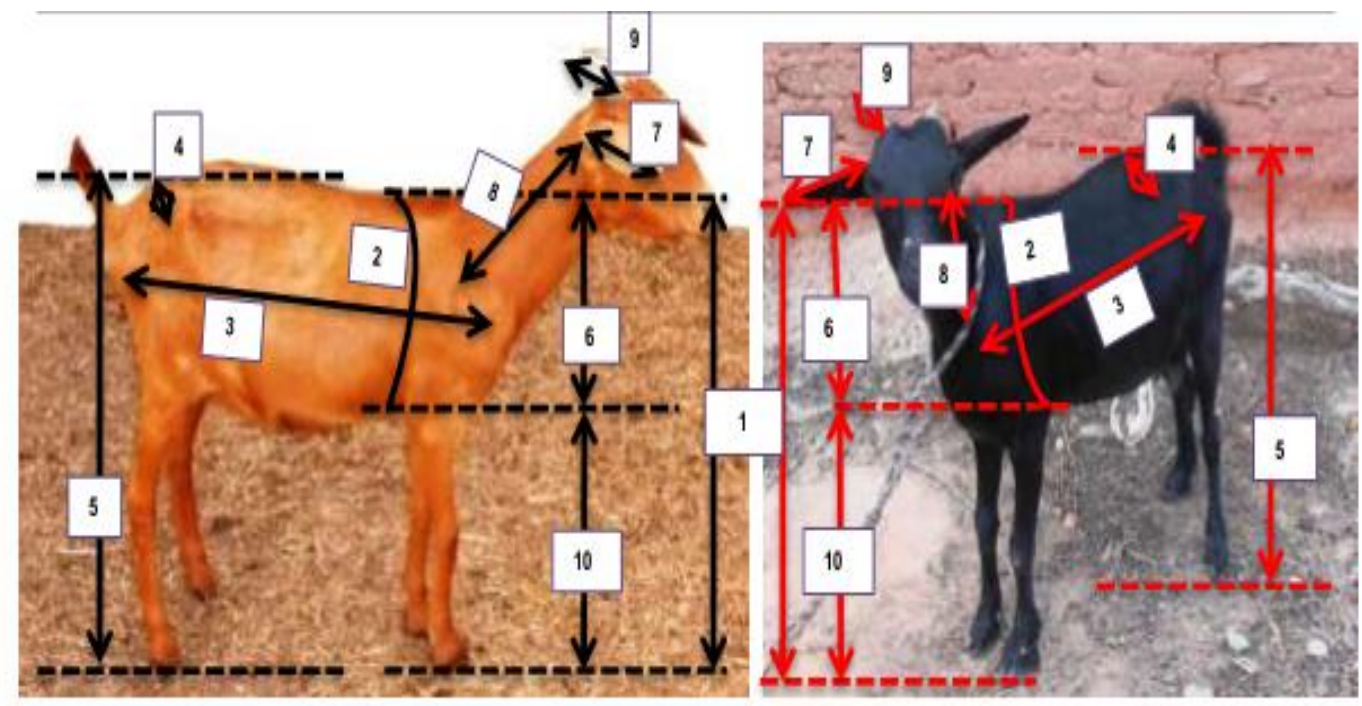

Figure 2: Positions des paramètres quantitatifs mesurés (Crédit photo: Adam Kadé M. G. et Mani M., 2018). 1 = Hauteur au Garrot; 2 = Tour de Poitrine; 3 = Longueur du Corps; 4 = Largeur du Bassin; 5 = Hauteur à la Croupe; $6=$ Profondeur de Poitrine; 7 = Longueur des Oreilles; 8 = Longueur du Cou; $9=$ Longueur des Cornes; $10=$ Vide Sous Sternal.

\section{RESULTATS}

\section{Paramètres qualitatifs}

Le Tableau 2 présente les résultats de l'analyse descriptive des paramètres qualitatifs. Ces résultats indiquent que, quels que soient le sexe et la couleur de la robe, la chèvre Rousse et ses variantes (Noires et Composées) ont présenté un type d'oreille dressée horizontalement $(73,9 \%$; $83,5 \%$; $87,5 \% ; 77,3 \%$ et $66,7 \%$ respectivement chez les mâles, les femelles, les animaux à robe rousse, les animaux à robe noire et les animaux à robe composée) et un profil de tête rectiligne $(100,0 \%)$. Le port de cornes a été majoritairement de type ibex chez les mâles $(69,6 \%)$ et chez les caprins Roux $(61,1 \%)$ tandis qu'il est de type markhar chez les femelles $(54,2 \%)$ et les Noirs $(63,1 \%)$. La différence des ports de cornes entre les deux sexes a été statistiquement significative ( $\mathrm{p}<$ 0,05 ). La barbiche était portée aussi bien par les mâles $(100,0 \%)$ que certaines femelles $(29,7 \%)$, la différence entre les deux sexes a été statistiquement significative. Les mâles toutes robes confondues ne portaient pas de pampilles $(100,0 \%)$, le port chez les femelles a été négligeable $(3,3 \%)$. Quels que soient le sexe et la couleur de la robe, les animaux ont présenté en moyenne des poils ras $(88,2 \%)$ et lisses $(95,8 \%)$. Le profil de la tête était rectiligne $(100,0 \%)$ chez tous les animaux. Les patrons pigmentaires principalement retrouvés étaient eumélanique noir $(43,1 \%$ de l'échantillon et $93,9 \%$ des Noirs), chocolat ( $39,6 \%$ de l'échantillon et $77,8 \%$ des Roux), phaeomélanique $(11,1 \%$ de l'échantillon et $22,2 \%$ des Roux). Il a été retrouvé en très faible proportion les patrons pigmentaires eumélanique uniforme et feu à ventre clair, chamoisé, mantelé et sauvage. Quelques rares cas de frosting $(1,4 \%)$ et de panachure (généralisée à $0,7 \%$ et localisée à $6,9 \%$ ) ont été relevés. Il a été noté une corrélation significative entre les modalités des variables qualitatives étudiées selon le sexe et la couleur de robe (Figure 3 ). Ces résultats montrent que les chèvres Rousse et Noire présentent les mêmes caractéristiques morphologiques à quelques exceptions près notamment liées au patron pigmentaire (eumélanique chocolat et phaeomélanique pour les chèvres Rousses et eumélanique noir pour les chèvres Noires) et le port des cornes (majoritairement ibex pour les Rousses et markhar pour les Noires). 


\section{Paramètres quantitatifs}

Les résultats de l'analyse descriptive des paramètres quantitatifs (moyennes, valeurs extrêmes et cumuls) selon le sexe et la couleur de la robe sont présentés dans le Tableau 3. Il ressort des résultats du test-t (Tableau 3) que, les moyennes de tous les paramètres quantitatifs ne sont pas statistiquement différentes selon le sexe $(\mathrm{p}>0,05)$ à l'exception de la Profondeur de Poitrine (PP), la Longueur des Oreilles (LO), la Longueur du Cou (LCU) et la Longueur des Cornes (LCR). La PP et la LCR sont plus importantes chez les mâles que les femelles et inversement pour LO et LCU.

L'analyse des variances a montré que la couleur de la robe n'a pas d'effet significatif sur les paramètres quantitatifs (ANOVA, $p>0,05$ ) sauf sur la Longueur des Cornes $(p=0,024)$.

L'analyse en composantes principales (ACP) a permis d'apprécier les corrélations entre toutes les variables quantitatives retenues en variables principales et entre elles et le sexe et la couleur de la robe en variables supplémentaires. Le Tableau 4 présente la matrice de corrélation de l'ACP. Celle-ci montre qu'il existe une corrélation significative $(p>0,05)$ entre les différents paramètres quantitatifs quelle que soit la couleur de la robe sauf la Longueur de l'Oreille (LO) qui n'est corrélée qu'à la Longueur du Cou (LCU). Il a été observé que le sexe a un effet significatif sur la LCR (positive avec le mâle et négative avec la femelle). Par ailleurs, la robe rousse a été corrélée parfaitement à la robe noire $\quad(\mathrm{r}=$ -0,92). Entre les deux sexes, la corrélation a été également parfaite $(\mathrm{r}=-1)$.

La Figure 4 présente la projection des individus étudiés sur le plan factoriel (F1, F2). Cette projection des individus sur le plan factoriel (F1, F2) en fonction de tous les paramètres quantitatifs étudiés rassemble la presque totalité des individus des trois groupes (roux, noirs, et composés) en un seul.

Tableau 2: Paramètres qualitatifs selon le sexe et la couleur de la robe de l'animal: fréquences en \% (effectifs) des modalités.

\begin{tabular}{|c|c|c|c|c|c|c|c|c|}
\hline \multirow{2}{*}{ Variable } & \multirow{2}{*}{ Modalité } & \multicolumn{2}{|c|}{ Sexe de l'animal } & \multirow{2}{*}{ Total } & \multicolumn{3}{|c|}{ Couleur de la robe } & \multirow{2}{*}{ Total } \\
\hline & & $\mathbf{M}^{*}$ & $\mathbf{F} * *$ & & Rousse & Noire & Composée & \\
\hline \multirow{3}{*}{ Type de l'oreille } & dressées & $73,9(17)$ & $83,5(101)$ & $81,9(118)$ & $87,5(63)$ & $77,3(51)$ & $66,7(4)$ & $81,9(118)$ \\
\hline & pédoncul & $13,0(3)$ & $12,4(15)$ & $12,5(18)$ & $8,3(6)$ & $16,7(11)$ & $16,7(1)$ & $12,5(18)$ \\
\hline & tombantes & $13,0(3)$ & $4,1(5)$ & $5,5(8)$ & $4,2(3)$ & 61(4) & $16,7(1)$ & $5,6(8)$ \\
\hline \multirow{2}{*}{ Type de cornes } & ibex & $69,6(16)^{\mathrm{a}}$ & $45,8(55)^{\mathrm{b}}$ & $49,6(71)$ & $61,1(44)$ & $36,9(24)$ & $50(3)$ & $49,7(71)$ \\
\hline & autres & $30,4(7)^{\mathrm{a}}$ & $54,2(65)^{\mathrm{b}}$ & $50,3(72)$ & $38,9(28)$ & $63,1(41)$ & $50(3)$ & $50,3(72)$ \\
\hline \multirow{3}{*}{ Port de barbiche } & présence & 100,0 & $29,7(36)^{b}$ & $41,0(59)$ & $45,8(33)$ & $33,3(22)$ & $66,7(4)$ & $41,0(59)$ \\
\hline & & $(23)^{\mathrm{a}}$ & & & & & & \\
\hline & absence & $0,0(0)^{\mathrm{a}}$ & $70,2(85)^{b}$ & $59,0(85)$ & $54,2(39)$ & $66,7(44)$ & $33,3(2)$ & $59,0(85)$ \\
\hline \multirow{2}{*}{$\begin{array}{l}\text { Port de } \\
\text { pampilles }\end{array}$} & présence & $0,0(0)$ & $3,3(4)$ & $2,8(4)$ & $1,4(1)$ & $4,5(3)$ & $0,0(0)$ & $2,8(4)$ \\
\hline & absence & $100,0(23)$ & $96,7(117)$ & $97,2(140)$ & $98,6(71)$ & $95,5(63)$ & $100,0(6)$ & $97,2(140)$ \\
\hline \multirow{3}{*}{$\begin{array}{l}\text { Longueur de } \\
\text { poils }\end{array}$} & ras & $91,3(21)$ & $87,6(106)$ & $88,2(127)$ & $90,3(65)$ & $86,4(57)$ & $83,3(5)$ & $88,2(127)$ \\
\hline & mi-long & $8,7(2)$ & $9,9(12)$ & $9,7(14)$ & $8,3(6)$ & $10,6(7)$ & $16,7(1)$ & $9,7(14)$ \\
\hline & long & $0,0(0)$ & $2,5(3)$ & $2,1(3)$ & $1,4(1)$ & $3,0(2)$ & $0,0(0)$ & $2,1(3)$ \\
\hline \multirow{2}{*}{ Type de poils } & lisse & $100,0(23)$ & $95,0(115)$ & $95,8(138)$ & $94,4(68)$ & $98,5(65)$ & $83,3(5)$ & $95,8(138)$ \\
\hline & non lisse & $0,0(0)$ & $5,0(6)$ & $4,2(6)$ & $5,6(4)$ & $1,5(1)$ & $16,7(1)$ & $4,2(6)$ \\
\hline Profil de la tête & rectiligne & $100,0(23) 1$ & $100,0(121)$ & $100,0(144)$ & $100,0(72)$ & $100,0(66)$ & $100,0(6)$ & $00,0(144)$ \\
\hline $\begin{array}{l}\text { Patron } \\
\text { pigmentaire }\end{array}$ & $\begin{array}{l}\text { eumélanique } \\
\text { noir }\end{array}$ & $26,1(6)$ & $46,3(56)$ & $43,1(62)$ & $0,0(0)$ & $93,9(62)$ & $0,0(0)$ & $43,1(62)$ \\
\hline
\end{tabular}




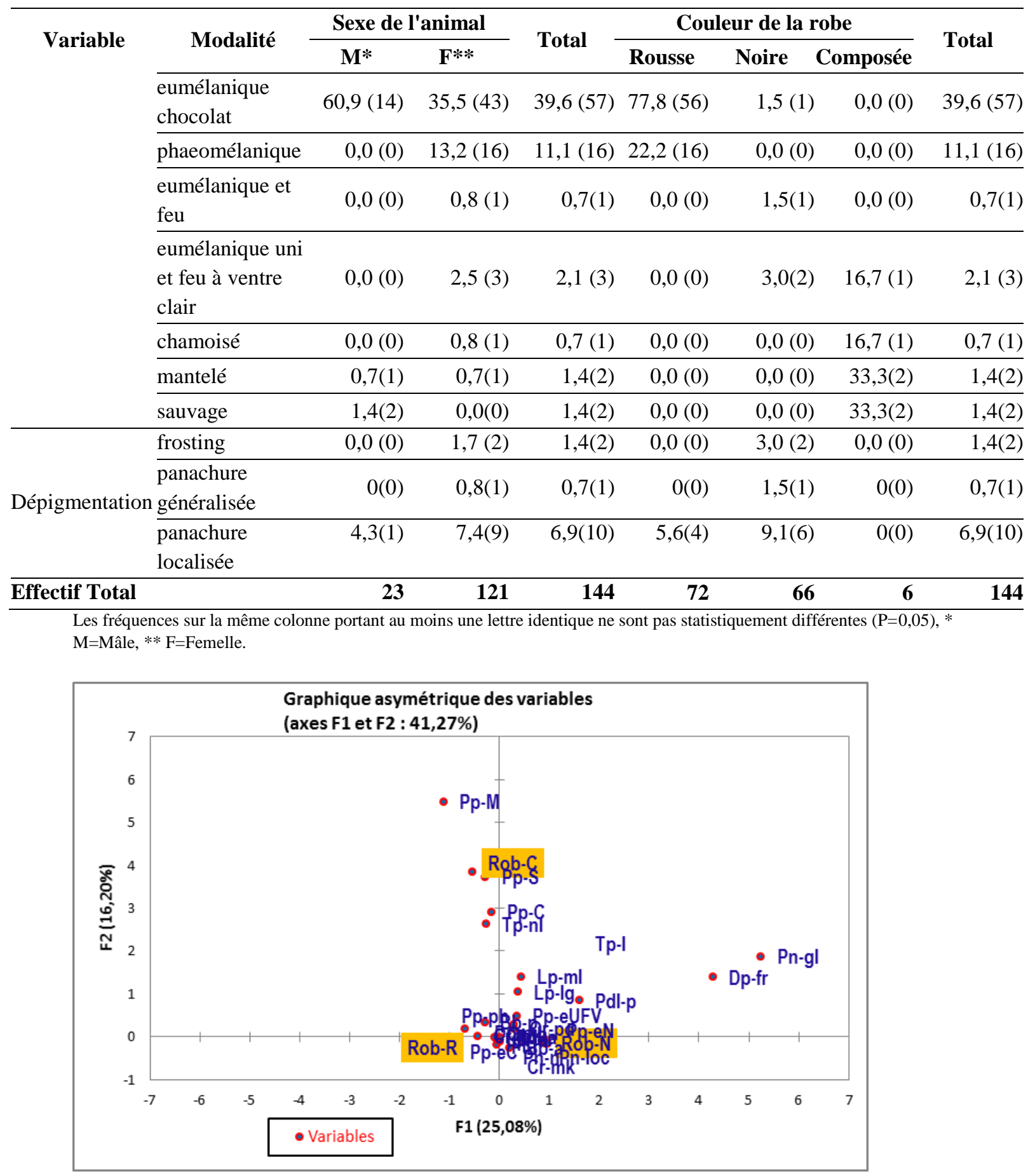

Figure 3: Carte factorielle des modalités des variables qualitatives. 
Tableau 4: Corrélations entre les différents paramètres quantitatifs des caprins selon le sexe et la couleur de robe.

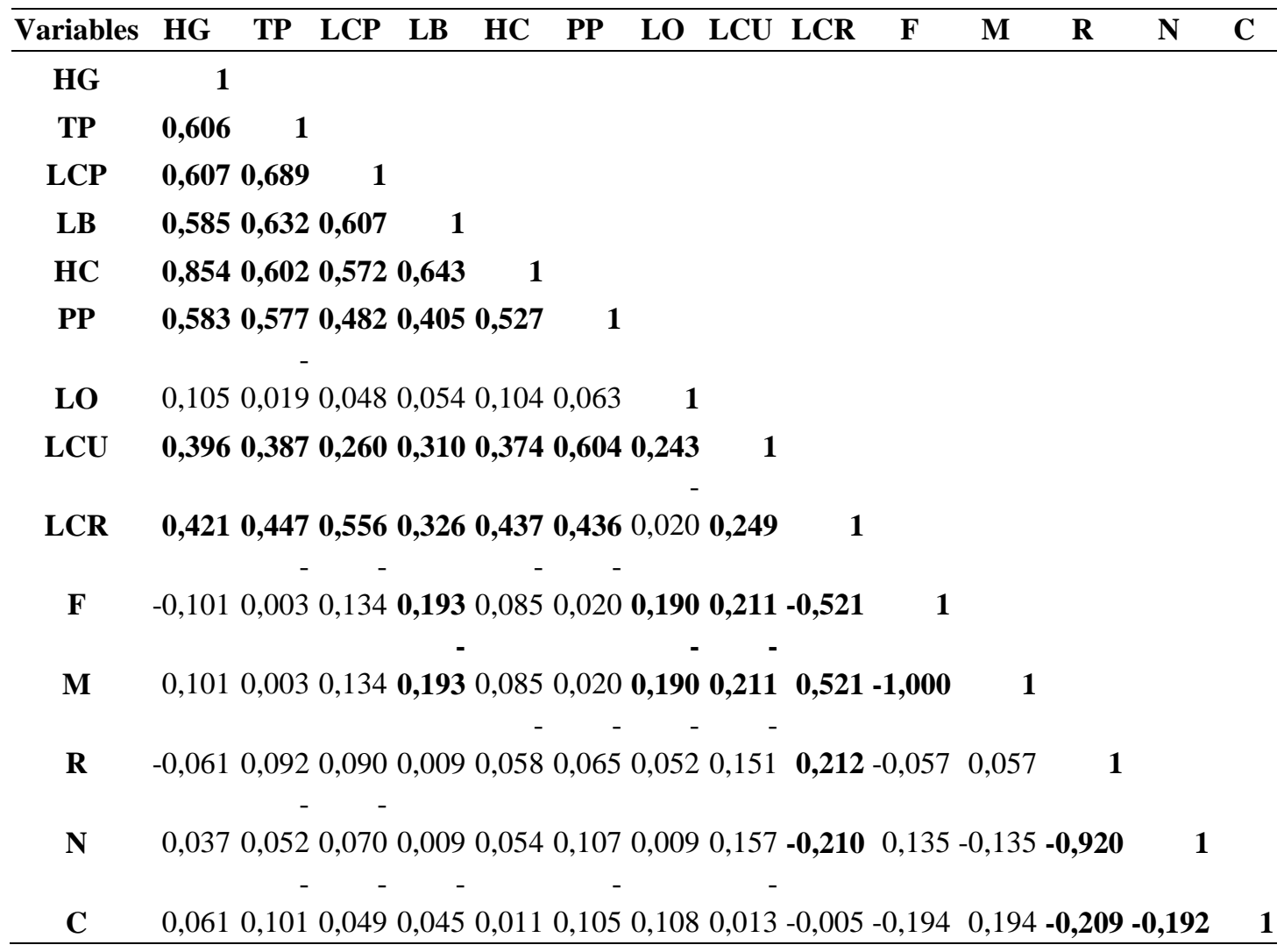

$\mathrm{F}=$ Femelle; $\mathrm{M}=$ Mâle; $\mathrm{R}=$ Rousse; $\mathrm{N}=$ Noire; $\mathrm{C}=$ Composée; HG = Hauteur au Garrot; TP = Tour de Poitrine; LCP = Longueur du Corps; LB = Largeur du Bassin; $\mathrm{HC}=$ Hauteur à la Croupe; $\mathrm{PP}=$ Profondeur de Poitrine; $\mathrm{LO}=$ Longueur des Oreilles; LCU = Longueur du Cou; LCR = Longueur des Cornes.

\section{DISCUSSION}

La présente étude vise à comparer les caractéristiques morphobiométriques aussi bien quantitatives que qualitatives de la chèvre Rousse de Maradi et sa variante Noire vivant dans la même zone agro-écologique. Il ressort de cette étude que certains paramètres qualitatifs sont caractéristiques de la chèvre Rousse. En effet, la chèvre Rousse possède majoritairement des oreilles dressées $(87,9 \%)$, des cornes ibex $(61,1 \%)$, des poils ras $(90,3 \%)$ et n'a pas de pampilles $(98,6 \%)$. Ces résultats, bien que légèrement différents de ceux obtenus par Karimou (2015) sur la même chèvre respectivement $98,5 \%, 75,8 \%, 96,8 \%$ et $97,6 \%$ suivent la même tendance.
Les patrons pigmentaires de la chèvre Rousse (chocolat à $77,8 \%$ ou phaeomélanique à $22,2 \%$ ), se distinguent nettement de ceux de la chèvre Noire (eumélanique noir à 93,9\%). En revanche, la similitude chez les deux types de chèvre réside dans l'aspect rare de la dépigmentation et de panachures.

Les valeurs de plusieurs paramètres quantitatifs obtenues sur la chèvre Rousse dans ce travail se rapprochent de celles rapportées par Karimou (2015); il s'agit du TP $(67,99 \mathrm{~cm} \pm 5,86$ contre $(v s) 68,40 \mathrm{~cm} \pm 6,41)$, la PP $(22,83 \mathrm{~cm} \pm 2,74$ vs $28,85 \mathrm{~cm} \pm 2,21)$, la LO $(13,15 \mathrm{~cm} \pm 1,11$ vs $12,81 \mathrm{~cm} \pm 1,39)$ et la LCR $(11,54 \mathrm{~cm} \pm 4,92$ vs $10,76 \mathrm{~cm} \pm 3,43)$. 
Tableau 3: Valeurs des paramètres quantitatifs selon le sexe et la couleur de la robe.

\begin{tabular}{|c|c|c|c|c|c|c|c|c|c|c|c|}
\hline \multirow{2}{*}{ Variables } & & \multirow{2}{*}{$\mathbf{N}$} & \multicolumn{9}{|c|}{ Les paramètres barymétriques selon le sexe et la couleur de la robe de l'animal } \\
\hline & & & HG & TP & $\mathbf{L C P}$ & $\mathbf{L B}$ & $\mathbf{H C}$ & $\mathbf{P P}$ & LO & $\mathbf{L C U}$ & LCR \\
\hline \multicolumn{12}{|c|}{ Selon le sexe } \\
\hline \multirow{2}{*}{ Mâle } & Moyenne & \multirow{2}{*}{23} & $60,09 \pm 4,24^{\mathrm{a}}$ & $67,52 \pm 5,87^{\mathrm{a}}$ & $63,39 \pm 6,85^{\mathrm{a}}$ & $11,09 \pm 1,54^{\mathrm{a}}$ & $62,74 \pm 4,09^{\mathrm{a}}$ & $23,13 \pm 2,80^{\mathrm{ab}}$ & $12,70 \pm 1,02^{\mathrm{ab}}$ & $23,04 \pm 3,04^{\mathrm{ab}}$ & $15,87 \pm 6,07^{\mathrm{ab}}$ \\
\hline & Extrema (Max; Min) & & 67,$0 ; 52,0$ & 78,$0 ; 56,0$ & 76,$0 ; 48,0$ & 14,$0 ; 8,0$ & 69,$0 ; 54,0$ & 30,$0 ; 18,0$ & 15,$0 ; 11,0$ & 32,$0 ; 19,0$ & 29,$0 ; 8,0$ \\
\hline \multirow{3}{*}{ Femelle } & Moyenne & \multirow{2}{*}{121} & $58,96 \pm 4,06^{\mathrm{a}}$ & $67,47 \pm 5,46^{\mathrm{a}}$ & $61,33 \pm 5,39^{\mathrm{a}}$ & $12,01 \pm 1,46^{\mathrm{a}}$ & $61,82 \pm 3,97^{\mathrm{a}}$ & $22,98 \pm 2,67^{\mathrm{ab}}$ & $13,31 \pm 1,20^{\mathrm{ab}}$ & $25,03 \pm 3,46^{\mathrm{ab}}$ & $9,61 \pm 3,19^{\mathrm{ab}}$ \\
\hline & Extrema (Max; Min) & & 68,$0 ; 48,0$ & 85,$0 ; 57,0$ & 79,$0 ; 50,0$ & 16,$0 ; 8,0$ & 70,$0 ; 51,0$ & 30,$0 ; 18,0$ & 16,$0 ; 10,0$ & 36,$0 ; 19,0$ & 20,$0 ; 0,0$ \\
\hline & Valeurs de $\mathrm{P}$ & & 0,248 & 0,713 & 0,184 & 0,328 & 0,859 & 0,014 & 0,008 & 0,008 & 0,000 \\
\hline \multirow{2}{*}{$\begin{array}{c}\text { Total tous sexes } \\
\text { confondus }\end{array}$} & s Moyenne & \multirow{2}{*}{144} & $59,14 \pm 4,10$ & $67,48 \pm 5,50$ & $61,66 \pm 5,67$ & $11,81 \pm 1,52$ & $61,97 \pm 3,99$ & $23,01 \pm 2,68$ & $13,22 \pm 1,20$ & $24,72 \pm 3,46$ & $10,6 \pm 4,4$ \\
\hline & Extrema (Max ; Min) & & 68,$0 ; 48,0$ & 85,$0 ; 56,0$ & 79,$0 ; 48,0$ & 16,$0 ; 8,0$ & 70,$0 ; 51,0$ & 30,$0 ; 18,0$ & 16,$0 ; 10,0$ & 36,$0 ; 19,0$ & 29,$0 ; 0,0$ \\
\hline \multicolumn{12}{|c|}{ Selon la couleur de la robe } \\
\hline \multirow{2}{*}{ Rousse } & Moyenne & \multirow{2}{*}{72} & $58,89 \pm 4,21^{\mathrm{a}}$ & $67,99 \pm 5,86^{\mathrm{a}}$ & $62,17 \pm 6,18^{\mathrm{a}}$ & $11,91 \pm 1,60^{\mathrm{a}}$ & $61,74 \pm 4,25^{\mathrm{a}}$ & $22,83 \pm 2,74^{a}$ & $13,15 \pm 1,11^{\mathrm{a}}$ & $24,19 \pm 3,42^{\mathrm{a}}$ & $11,54 \pm 4,92^{\mathrm{ab}}$ \\
\hline & $\overline{\text { Extrema (Max; Min) }}$ & & 67,$0 ; 52,0$ & 85,$0 ; 57,0$ & 79,$0 ; 48,0$ & 16,$0 ; 8,0$ & 70,$0 ; 52,0$ & 30,$0 ; 18,0$ & 16,$0 ; 10,0$ & 36,$0 ; 19,0$ & 29,$0 ; 2,0$ \\
\hline \multirow{2}{*}{ Noire } & Moyenne & \multirow{2}{*}{66} & $59,30 \pm 4,09^{\mathrm{a}}$ & $64,84 \pm 5,00^{\mathrm{a}}$ & $61,23 \pm 5,07^{\mathrm{a}}$ & $11,67 \pm 1,42^{\mathrm{a}}$ & $62,20 \pm 3,82^{\mathrm{a}}$ & $23,32 \pm 2,70^{\mathrm{a}}$ & $13,23 \pm 1,27^{\mathrm{a}}$ & $25,30 \pm 3,54^{\mathrm{a}}$ & $9,61 \pm 3,73^{\mathrm{ab}}$ \\
\hline & Extrema (Max; Min) & & 68,$0 ; 48,0$ & 76,$0 ; 58,0$ & 76,$0 ; 50,0$ & 14,$0 ; 8,0$ & 70,$0 ; 51,0$ & 30,$0 ; 18,0$ & 16,$0 ; 11,0$ & 33,$0 ; 19,0$ & 25,$0 ; 0,0$ \\
\hline \multirow{3}{*}{ Composée } & Moyenne & \multirow{2}{*}{6} & $60,33 \pm 2,94^{\mathrm{a}}$ & $64,83 \pm 6,34^{\mathrm{a}}$ & $60,33 \pm 5,89$ & $11,33 \pm 1,51^{\mathrm{a}}$ & $62,17 \pm 2,79^{\mathrm{a}}$ & $21,67 \pm 0,52^{\mathrm{a}}$ & $13,83 \pm 1,33^{\mathrm{a}}$ & $24,50 \pm 2,26^{\mathrm{a}}$ & $10,50 \pm 2,59^{\mathrm{ab}}$ \\
\hline & Extrema (Max; Min) & & 64,$0 ; 56,0$ & 73,$0 ; 56,0$ & 70,$0 ; 53,0$ & 13,$0 ; 9,0$ & 66,$0 ; 58,0$ & 22,$0 ; 21,0$ & 15,$0 ; 12,0$ & 28,$0 ; 22,0$ & 14,$0 ; 8,0$ \\
\hline & Valeurs de $\mathrm{P}$ & & 0,382 & 0,464 & 0,424 & 0,632 & 0,760 & 0,407 & 0,203 & 0,194 & 0,024 \\
\hline \multirow{2}{*}{$\begin{array}{l}\text { Total toutes } \\
\text { robes } \\
\text { confondues }\end{array}$} & Moyenne & \multirow[b]{2}{*}{144} & $59,14 \pm 4,10$ & $67,48 \pm 5,50$ & $61,66 \pm 5,67$ & $11,81 \pm 1,52$ & $61,97 \pm 3,99$ & $23,01 \pm 2,68$ & $13,22 \pm 1,20$ & $24,72 \pm 3,46$ & $10,61 \pm 441$ \\
\hline & Extrema (Max ; Min) & & 68,$0 ; 48,0$ & 85,$0 ; 56,0$ & 79,$0 ; 48,0$ & 16,$0 ; 8,0$ & 70,$0 ; 51,0$ & 30,$0 ; 18,0$ & 16,$0 ; 10,0$ & 36,$0 ; 19,0$ & 29,$0 ; 0,0$ \\
\hline
\end{tabular}

Les moyennes sur la même colonne portant au moins une lettre identique ne sont pas statistiquement différentes $(\mathrm{P}=0,05)$. HG = Hauteur au Garrot; TP = Tour de Poitrine; LCP = Longueur du Corps; LB = Largeur du Bassin; HC = Hauteur à la Croupe;

$\mathrm{PP}=$ Profondeur de Poitrine; $\mathrm{LO}=$ Longueur des Oreilles; $\mathrm{LCU}=$ Longueur du Cou; $\mathrm{LCR}=$ Longueur des Cornes. 


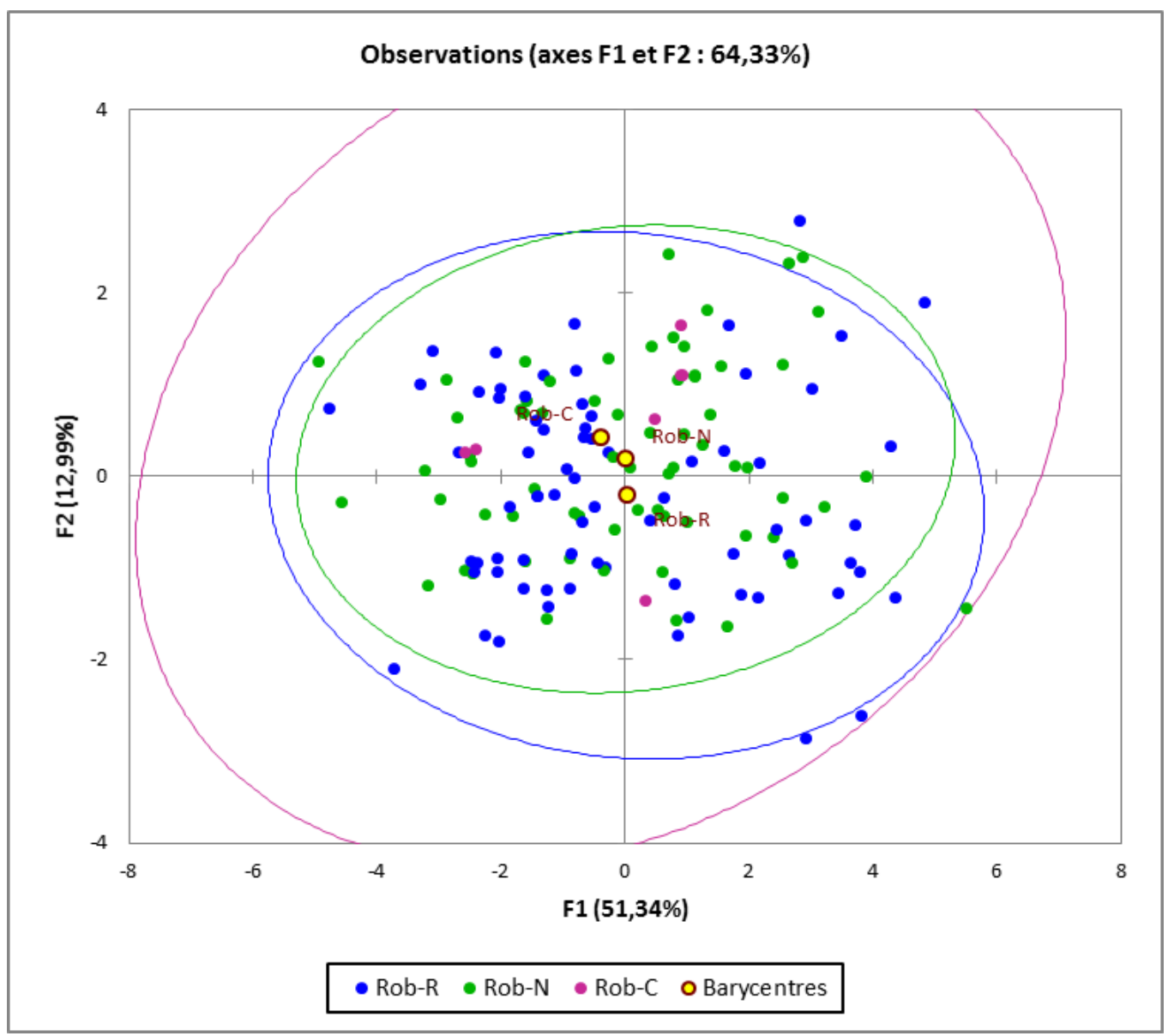

Figure 4: Carte factorielle des individus.

Les valeurs moyennes de la hauteur au garrot obtenues sur les chèvres Rousse et Noire de Maradi sont respectivement $58,89 \mathrm{~cm} \pm 4,21$ et $59,25 \mathrm{~cm} \pm 4,05$. Ces valeurs sont inférieures à celles obtenues sur la chèvre Rousse par Robinet (1967); Marichatou et al. (2002); Karimou (2015) qui sont respectivement: $62-67 \mathrm{~cm}, 63,10 \mathrm{~cm} \pm 5,18$ et $64,9 \mathrm{~cm}$ au Niger dans la région de Maradi et environs. La même tendance sur la chèvre Rousse a été observée par Abdulmojeed et al. (2011) dans les Etats de Sokoto, Kaduna et Kano au Nigeria $(62,34 \mathrm{~cm} \pm 0,51)$. Alphonsus et al. (2010) ont également relevé des résultats similaires en ce qui concerne la
LCP, le TP et la HG sur la chèvre Rousse à Kano.

Les valeurs obtenues sont par contre légèrement supérieures à celles obtenues par Ngona et al. (2012) sur la chèvre de Lubumbashi $(53 \mathrm{~cm})$ en République Démocratique du Congo, la chèvre de Mashona au Zimbabwe $(56,7 \mathrm{~cm})$ et la chèvre West African Dwarf au Bénin (48,2 cm). Ces valeurs dépassent également celles enregistrées au Burkina Faso $(48,4 \mathrm{~cm})$ sur la chèvre de type Mossi par Traoré et al. (2006). La couleur de la robe distingue à première vue les chèvres Rousse et Noire de Maradi mais pour beaucoup de paramètres quantitatifs, les valeurs moyennes en $\mathrm{cm}$ ne 
sont pas statistiquement différentes $(\mathrm{p}>0,05)$ comme présenté dans le Tableau 3. Des observations similaires avaient été faites par Marichatou et al. (2002) sur les chèvres Rousses et Noires en milieu villageois dans trois départements (Guidan Roumdji, Madarounfa et Tessaoua). Quant à la chèvre à robe composée, elle semble être proche des deux précédentes à travers les moyennes des paramètres quantitatifs étudiés.

\section{Conclusion}

Le présent travail a montré que les deux types de caprins (chèvres Rousse et Noire) présentent des caractères quantitatifs statistiquement similaires. De même, tous les caractères qualitatifs rapprochent les deux types de caprins, exception faite de la panachure, de la dépigmentation et du type de poils. En définitive, il est ressorti de cette étude qu'en réalité, les robes rousses et noires constituent une diversité dans la même race caprine. Lors des diffusions des chèvres par certains projets de développement et organisations non gouvernementales en vue d'assurer la résilience des populations vulnérables ou atteindre l'autonomisation des femmes rurales, les chèvres de couleur noire ne doivent pas faire l'objet de discrimination.

Ces résultats devront être complétés par des données issues d'une caractérisation moléculaire et l'étude des paramètres zootechniques pour éclairer davantage la similitude entre ces deux «variantes» de la chèvre de Maradi.

\section{CONFLIT D'INTERETS}

Les auteurs déclarent qu'il n'y a aucun conflit d'intérêts entre les différents contributeurs.

\section{CONTRIBUTIONS DES AUTEURS}

Dans le cadre de ce travail, chaque auteur a apporté sa contribution selon l'état d'avancement. MGAK a participé à la collecte, à l'analyse statistique et à la rédaction de cet article. MM a parrainé la collecte ainsi que l'analyse statistique des données et a contribué à la rédaction. G-KD a pris part à l'harmonisation des données et à la perfection de la rédaction. HM a dirigé l'ensemble des travaux.

\section{REMERCIEMENTS}

Les auteurs remercient M. Moussa Keita, Directeur Général des Centres de Multiplication du Bétail, M. Nouhou Moussa, Directeur du Centre Caprin de Maradi, Dr Mahamane Amadou Soumaïla, Directeur Régional de l'Elevage de Maradi et M. Nouri Brah, Chercheur au Centre Régional de Recherche Agronomique de Maradi, pour leur facilitation au cours de ce travail. Que toutes les populations des villages de collecte de données, l'ensemble du personnel et stagiaires des services d'Elevage des départements d'Aguié, Guidan Roumdji, Madarounfa et Tessaoua ainsi que celui de la Direction Régionale de l'Elevage de Maradi soient remerciés.

\section{REFERENCES}

Abdulmojeed Y, Adebowale ES, Ikhide GI. 2011. Comparative multivariate analysis of biometric traits of West African Dwarf and Red Sokoto goats. Trop. Anim. Health Prod., 43(3): 561-566. DOI: 10.1007/s11250-010-9731-y.

Adigwe PI, Fayemi O. 2005. A biometric study of the reproductive tract of the Red Sokoto (Maradi) goats of Nigeria. Pak. Vet. J., 25 (3): 149-150.

Alphonsus C, Akpa GN, Sam IM, Agubos OCP, Finangwai FI, Mukasa C. 2010. Relationship of parity and some breeding characteristics in Red Sokoto goats. Cont. J. An. and Vet. Res., 2: 25-30. http://www.wiloludjournal.com. 
AU-IBAR. 2019. Visual field teaching aid/ documentary for phenotypic and morphometric characterization of animal genetic resources. AU-IBAR.

Bembello A. 1961. La chèvre Rousse et son exploitation au Niger. Thèse de doctorat de l'ecole de médecine vétérinaire de Toulouse, France.

Blench R. 1999. Traditional livestock breeds: Geographical distribution and dynamics in relation to the ecology of West Africa. Overseas Development Institute Portland House Stag Place London, SW1E 5DP, $69 \mathrm{p}$.

Charray YC, Haumesser J, Planchenault D. 1980. Synthèse des connaissances sur l'élevage des petits ruminants dans les pays tropicaux d'Afrique Centrale et d'Afrique de l'Ouest. Institut d'Elevage et de Médecine Vétérinaire des pays tropicaux, Paris, 295 p.

Châtelain E. 1987. Atlas d'anatomie de la chèvre, Capra hircus L. ENV Lyon, Marcy-l'Etoile-69752 Charbonnières Cedex : Lyon, France ; 203 p.

Djariri B. 2006. Etude sur la monographie de la chèvre rousse de Maradi. Consultation pour le Ministère des Ressources Animales du Niger, $50 \mathrm{p}$.

Rischkowsky B, Pilling D. 2008. L'état des ressources zoogénétiques pour l'alimentation et l'agriculture dans le Rome. FAO, 556 P.

FAO. 2013. Caractérisation phénotypique des ressources génétiques animales. Directives FAO sur la production et la santé animales $\mathrm{N}^{\circ} 11$. FAO - Rome, 151 p.

Hassan A, Ciroma A. 1988. Body weight measurements relationship in Nigerian Red Sokoto goats - Relations entre le poids vif et les mensurations du corps chez la chèvre rousse de Maradi.
Karimou B. 2015. Caractérisation phénotypique et zootechnique de la chèvre rousse de Maradi. Thèse de Doctorat, Université Abdou Moumouni de Niamey, $142 \mathrm{p}$.

Labbo M. 1999. Contribution à l'étude comparative des performances zootechniques de la Chèvre Rousse de Maradi et de son «Apparentée» à robe noire dans la zone de Maradi/Niger. Mémoire de fin d'étude, Université du Mali, Institut polytechnique rural de formation et de recherche appliquée, IPR/IFRA, Bamako, Mali, 46 p.

Mani M, Marichatou H, Mouiche M, Issa M, Chaïbou I, Sow A, Chaïbou M, Sawadogo JG. 2014. Caractérisation de la chèvre du sahel au Niger par analyse des indices biométriques et des paramètres phénotypiques quantitatifs. Anim. Genet. Resour., 54: 21-32. DOI: 10.1017/S2078633614000241

Mani M. 2013. Caractérisation phénotypique et zootechnique de la chèvre du sahel élevée au Niger. Thèse pour l'obtention du grade de Docteur à l'Université Abdou Moumouni de Niamey, Niger, $190 \mathrm{p}$.

Marichatou H, Karimou B, Issa M, Chaibou M, Banoin M, Yénikoye A, Falké DI, Ayatundé A. 2012. Caractéristique morphologique de la chèvre rousse du Niger. Anim. Genet. Resour., 51: 89-97. DOI: https://doi.org/10.1017/S20786336 12000458

Marichatou H, Mamane L, Banoin M, Baril G. 2002. Performances zootechniques des caprins du Niger: étude comparative de la Chèvre Rousse de Maradi et de la chèvre à robe noire dans la zone de Maradi, Rev. Elev. Med. Vet. Pays Trop., 55(1): 79-84. DOI: https://doi.org/10.19182/remvt.9850 
Ngona IA, Beduin JM, Khang'Maté ABF, Hanzen C. 2012. Etude descriptive des caractéristiques morphométriques et génitales de la chèvre de Lubumbashi en République Démocratique du Congo. Rev. Elev. Med. Vet. Pays Trop., 65(3-4): 75-79.

DOI: https://doi.org/10.19182/remvt.101 26

Niger. 2013. Stratégie de Développement Durable de l'Elevage (SDDEL 20132035). SDDEL, Niger, 83 p.

Niger. 2014. Rapport national d'aide à la préparation du Deuxième Rapport sur l'État des Ressources Zoogénétiques pour l'Alimentation et l'Agriculture dans le Monde, incluant des données spécifiques au secteur servant pour l'État de la Biodiversité pour l'Alimentation et l'Agriculture dans le Monde. Niger, $54 \mathrm{p}$. Niger. 2016. Le Niger en chiffres. Institut National de la Statistique-Niger, $84 \mathrm{p}$.
Robinet AH. 1967. La chèvre rousse de Maradi; son exploitation et sa place dans l'économie et l'élevage de la République du Niger. Rev. Elev. Med. Vet. Pays Trop., 20 (1): 129-186.

Robinet AH. 1971. La chèvre de Maradi et l'élevage caprin au Niger. II $^{\text {ème }}$ Conférence Internationale sur l'élevage caprin. Tours, France, 19 p.

Roth M. 1938. La petite chèvre rousse du Niger. Bull. Serv. Zootech. Epizoot. Afr. Occid. Fr., 1(2): 13 - 19.

Traoré A, Tamboura HH, Kaboré A, Yaméogo N, Bayala B, Zaré I. 2006. Caractérisation morphologique des petits ruminants (ovins et caprins) de race locale 'Mossi', au Burkina Faso. Agri., 39: 39-50.

DOI: 10.1017/S1014233900002121 